\title{
An Amphiphilic Approach to Nanocrystal Quantum Dot-Titania Nanocomposites Supporting Information
}

\author{
Melissa A. Petruska*, Andrew P. Bartko, and Victor I. Klimov* \\ Preparation of NQD-titania sols
}

Polymer approach:

$40 \%$ octylamine-modified poly(acrylic acid) is prepared from poly(acrylic acid) with a molecular weight ranging from 1000-10,000 according to procedures described in Reference 1.

A solution of $40 \%$ octylamine-modified poly(acrylic acid) in chloroform is mixed with a solution of nanocrystal quantum dots (NQDs) in chloroform. When the appropriate amount of polymer is added (typically 1-2.5 times the mass of NQDs depending on NQD size and surface cap), the NQDs become soluble in ethanol. The chloroform is evaporated, and the NQDs are taken into the inert atmosphere glove box. Ethanol (and if necessary a small amount of THF) is added to the NQDs to solubilize them. Following the addition of titanium(IV) butoxide (1:1 NQD/titania mass ratio), the solution is allowed to stir for up to two hours at room temperature. It is then filtered through a 0.2 $\mu \mathrm{m}$ syringe filter and spin-coated onto the appropriate substrate. Volume fractions of NQDs as high as $6 \%$ are easily achieved and are measured according to procedures discussed in Reference 2. The titania films communicated in this work have similar properties to analogous titania materials discussed in Reference 2, which show excellent photostability for hours of illumination at intensities sufficient to produce optical gain.

Blank runs (no polymer added):

To prepare NQD-titania sols in which polymer was omitted, we use a 2:1 THF/ethanol ratio since the NQDs are not soluble in ethanol in their as-prepared, hydrophobic state. In a typical procedure, $0.15 \mathrm{~mL}$ THF and $0.075 \mathrm{~mL}$ ethanol are added to $20 \mathrm{mg}$ of CdSe NQDs in an inert atmosphere dry box. Once everything is in solution, $15 \mathrm{mg}$ of titanium(IV) butoxide is added, and the solution is allowed to stir at $50-60{ }^{\circ} \mathrm{C}$ for 5 hour. The solution is filtered, and films are prepared by spin-coating the sol onto the appropriate substrates.

References:

1. Wang, K. T.; Iliopoulos, I.; Audebert, R. Polymer Bull. 1988, $20,577$.

2. Petruska, M. A.; Malko, A. V.; Voyles, P. M.; Klimov, V. I. Adv. Mater. 2003, 15,610 